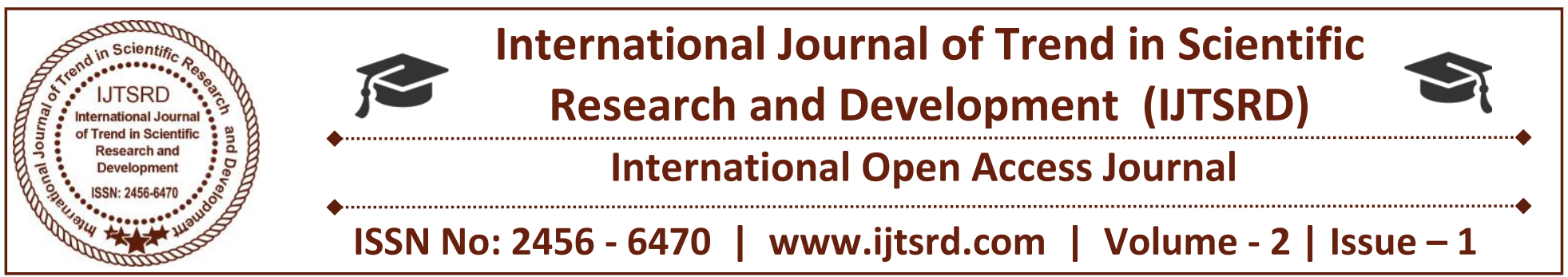

\title{
Scheduling Algorithms in Cloud Computing - An Extensive Survey
}

\author{
S. Saranya Devi \\ PG Scholor, Department Of CSE, \\ Anna University Regional \\ Campus, Coimbatore
}

\author{
V. Venkatesa Kumar \\ Assistant Professor, Department \\ Of CSE, Anna University Regional \\ Campus, Coimbatore
}

\author{
M. Newlin Rajkumar \\ Assistant Professor, Department \\ Of CSE, Anna University Regional \\ Campus, Coimbatore
}

\begin{abstract}
In any computing environment, the availability of resources is finite, so effective utilization of resources is very important. To achieve effective management of the available resources in a scalable environment optimal mechanism is required. In order to do this, choosing of scheduling algorithm is important. The scheduling techniques should be selected in such a way that the various user demands are satisfied. In this paper, an overview of different scheduling techniques like cost and energy aware scheduling, cloud based workflow scheduling, an improved ant colony algorithm in cloud computing environment are analysed.
\end{abstract}

Keywords: Cloud computing, Scheduling

\section{INTRODUCTION}

Cloud computing can be generally defined as computing services delivered to the user over the internet. Cloud computing is used to access the pooled resources required for computing through our browser's window. Cloud computing is defined in [7] which refers to applications and services that run on distributed network using virtualized resources and accessed by common Internet protocols and networking standards. Cloud computing is a promising computing paradigm, where a large pools of systems are connected in private or public networks, to provide automatically scalable infrastructure for data, application and file system. The five important characteristics of cloud computing are
1. On-demand self-service

2. Resource pooling

3. A broad network access

4. Rapid elasticity

5. Measured service

Cloud computing types are public, private, community and hybrid cloud. Public cloud is owned and operated by third parties; they deliver superior economics of scale to customers, as the infrastructure costs are covered among a mix of users, giving each of the separate client an attractive low-cost, "Pay-asyou-go" mode. Private clouds are of two types: Onpremise private clouds and externally hosted private cloud. Hybrid cloud is the combination of both public and private cloud models. This environment has the ability of providing on demand, externally provisional scale services. Cloud infrastructure is shared by several organizations and supports a specific community that has shared concerns in the community cloud. It may be handled by the organizations or a third party and may exist on premise or off premise. Cloud providers offer services that can be grouped into three categories. They are

1. Software as a Service (SaaS),

2. Platform as a Service(PaaS),

3. Infrastructure as a Service (IaaS).

Software as a Service (SaaS) is used by end users. Platform as a Service (PaaS) is used by a developer or programmer. Infrastructure as a Service (IaaS) is used by the system admin. Scheduling is one of the most prominent activities that executes in a cloud computing environment. Scheduling plays a major 
role in allocating resources for competing process. Scheduling is a method by which threads, either processes or data flows are given access to system resources. Normally scheduling can be carried out for load balancing in a system to achieve quality of service effectiveness. The need for a scheduling algorithm arises from the requirement of most modern systems to perform multitasking (execute more than one process at a time) and multiplexing (transmit multiple flows simultaneously). The scheduler is concerned mainly with throughput, latency, turnaround time, response time and fairness/ waiting time.

\section{RELATED WORK}

\subsection{WORKFLOWSSCHEDULING}

\section{Scientific Workflows Scheduling With Deadline} Constraint In The Clouds [1]: cloud computing has been commonly identified as the fundamental of computing paradigm to implement, compute and data intensive business process workflow and scientific workflow application for processing huge amount of scientific data. In cloud computing, there is an one important feature named Multi-tenanted. It provides scalability and economic advantages to final customers and service providers are shares the similar cloud platform. In multi-tenant cloud computing, resource management is becoming one of the biggest tasks because of inherent heterogeneity and resource isolation. In this multi-tenant cloud computing, cloud based workflow scheduling algorithm is used to compute intensive workflow applications. It helps to minimize the complete workflow completion time, tardiness, execution cost of the workflows and it mainly utilize free resources of cloud effectively. Cost of execution of workflow changes depending on the application and on the size of the workflow. In this four-layered workflow scheduling system is introduced. This proposed cloud based workflow scheduling algorithms contrasted with the state-ofthe-art algorithm that is First Come First Served, EASY Backfilling and minimum completion time scheduling policies to calculate the performance. Proposed algorithm compared with different scheduling algorithm to highlight the Performance and robustness of the proposed solution. Cloud based workflow scheduling algorithm scheduling performs are best when compared with other in the terms of cost by generating much cheaper schedules.
Cost And Energy Aware Scheduling Algorithm For Scientific Workflows With Deadline Constraint [2]:

To implement the deadline constrained scientific workflows, cloud computing is the best suitable platform. In which, there are big data applications and often need huge time to complete. In the clouds, energy consumption has become one of the main problems. So cost and energy aware scheduling (CEAS) algorithm is introduced by cloud scheduler. By using this algorithm, execution cost of workflow is minimizing and minimize the energy consumption while meeting the deadline constraint. In this proposed (CEAS) cost and energy aware scheduling comprise of five sub algorithm. Initially, the virtual machine selection algorithm is used to implement cost utility idea to direct task to their correct virtual machines (VM) types. Next, two tasks are merged by using merging methods to minimize cost of execution and energy consumption. In last task slack algorithm is used to save the energy by DVFS techniques. In other word, sequence tasks merging, parallel tasks merging and VM reuse algorithms will minimize the economic cost of workflow is energetic. In addition, sequence tasks merging, the parallel tasks merging, $\mathrm{VM}$ reuse and task slacking algorithm can protect respectable energy. The time complexity of each sub algorithm is polynomial. This cost and energy aware scheduling (CEAS) algorithm is estimated by using cloudsim and for real-world scientific workflow application is described.

\section{Hybrid Cloud Resources Scheduling Methods Based} On Deadline And Cost Constraints [3]: To optimize the fixed pool of public and private computing resources, task-oriented multi-objective scheduling method based on ant colony optimization (MOSACO) is introduced. According to deadline and cost constraints in hybrid cloud computing environment. Multi-objective scheduling method based on ant colony optimization (MOSACO) is applied to reduce task completion times and costs by using time-first and cost-first single objective optimization strategies. It is used to increase user quality of service and the profit of resource providers by working an entropy optimization model. The value of the multi-objective scheduling method based on ant colony optimization 
(MOSACO) algorithm based on various considerations of task Completion time, Cost, number of deadline violations, private resource utilization is evaluated by using simulation and three application instance. Comparisons process is done with a similar scheduling algorithm.

\subsection{TASK SCHEDULING}

\section{Temporal Task Scheduling For Profit Maximization}

In Hybrid Clouds [4]: As cloud computing is becoming increasingly popular, consumers' tasks around the world arrive in cloud data centers. Scheduling tasks while assuring the service delay bound of delay-tolerant tasks. A challenging problem is the aperiodicity of arrival tasks and how to dynamically schedule all arrival tasks given the fact that the capacity of a private cloud provider is limited. In Previous works, an admission control to intelligently refuse some of arrival tasks. Although, this will reduce the throughput of a private cloud, and affect revenue loss. The problem of how to increase the profit of a private cloud in hybrid clouds while assuring the service delay bound of delay-tolerant tasks. So a profit maximization algorithm(PMA) to find out the temporal variation of prices in hybrid clouds. The temporal task scheduling contributes by PMA can automatically schedule all arrival tasks to execute in private and public clouds. The sub problem in each iteration of the profit maximization algorithm (PMA) clarified by the proposed hybrid heuristic optimization algorithm, parallel annealing particle swarm optimization (SAPSO). Finally, the proposed method can greatly increase the throughput and the profit of a private cloud and energy aware scheduling comprise of five sub algorithm. Initially, the virtual machine selection algorithm is used to implement cost utility idea to direct task to their correct virtual machines (VM) types. Next, two tasks are merged by using merging methods to minimize cost of execution and energy consumption. In last task slack algorithm is used to save the energy by DVFS techniques. In other word, sequence tasks merging, parallel tasks merging and VM reuse algorithms will minimize the economic cost of workflow is energetic. In addition, sequence tasks merging, the parallel tasks merging,
VM reuse and task slacking algorithm can protect respectable energy. The time complexity of each sub algorithm is polynomial. This cost and energy aware scheduling (CEAS) algorithm is estimated by using cloudsim and for real-world scientific workflow application is described.

Hybrid Cloud Resources Scheduling Methods Based On Deadline And Cost Constraints [3]:To optimize the fixed pool of public and private computing resources, task-oriented multi-objective scheduling method based on ant colony optimization (MOSACO) is introduced. According to deadline and cost constraints in hybrid cloud computing environment. Multi-objective scheduling method based on ant colony optimization (MOSACO) is applied to reduce task completion times and costs by using time-first and cost-first single objective optimization strategies. It is used to increase user quality of service and the profit of resource providers by working an entropy optimization model. The value of the multi-objective scheduling method based on ant colony optimization (MOSACO) algorithm based on various considerations of task Completion time, Cost, number of deadline violations, private resource utilization is evaluated by using simulation and three application instance. Comparisons process is done with a similar scheduling algorithm.

\subsection{TASK SCHEDULING}

Temporal Task Scheduling For Profit Maximization In Hybrid Clouds [4]: As cloud computing is becoming increasingly popular, consumers' tasks around the world arrive in cloud data centers. Scheduling tasks while assuring the service delay bound of delay-tolerant tasks. A challenging problem is the aperiodicity of arrival tasks and how to dynamically schedule all arrival tasks given the fact that the capacity of a private cloud provider is limited. In Previous works, an admission control to intelligently refuse some of arrival tasks. Although, this will reduce the throughput of a private cloud, and affect revenue loss. The problem of how to increase the profit of a private cloud in hybrid clouds while assuring the service delay bound of delay-tolerant tasks. So a profit maximization algorithm(PMA) to 
find out the temporal variation of prices in hybrid clouds. The temporal task scheduling contributes by PMA can automatically schedule all arrival tasks to execute in private and public clouds. The sub problem in each iteration of the profit maximization algorithm (PMA) clarified by the proposed hybrid heuristic optimization algorithm, parallel annealing particle swarm optimization (SAPSO). Finally, the proposed method can greatly increase the throughput and the profit of a private cloud while guaranteeing the service delay bound.

\section{Joint Optimization of Operational Cost And} Performance Interference [5]: In a data center, Virtual machine (VM) scheduling is a technique for the efficient operation of the computing resources. Previous work has mainly focused on consolidating VMs to improve resource utilization and to optimize energy consumption. In spite, the interference between collocated VMs is usually ignored, which can result in much worse performance degradation of the applications running on the Virtual Machines due to the contention of the shared resources. Based on this observation, we aim at designing efficient Virtual Machines assignment and scheduling strategies in which we consider optimizing both the operational cost of the data center and the performance degradation of the running applications.so that finally proposed a general model that captures the tradeoff between the two contradictory objectives. There is an offline and online solutions for this problem by exploiting the spatial and temporal information about performance interference of Virtual Machines collocation, where Virtual Machines scheduling is performed by jointly considering the combinations and the life-cycle overlap of the Virtual Machines. Evaluation results show that the proposed methods can generate efficient schedules for Virtual Machines, achieving low operational cost reducing the performance degradation of applications in cloud data centers.

\section{Simultaneous Cost And Qos Optimization In Cloud} [6]: Cloud computing offers resources and services for Web applications. In cloud resource allocation, Selection of optimal cloud resources is the important goal. In some cases, cloud provider's pricing strategy is developed for the interest of the providers so customers pay more than needs. Nonetheless, cloud customers are interested in selecting cloud resources to meet their quality of service requirements. In the interest of both providers and customers, it is essential to balance the two conflicting objectives of deployment cost and Quality of Service performance. In this, present a cost-effective and a runtime friendly algorithm that reduces the deployment cost while meeting the Quality of Service performance requirements. In other words, the algorithm grant an optimal choice, from customers view, for deploying a Web application in a cloud computing environment. The multi-objective optimization algorithm cheapen cost and increases Quality of Service performance in parallel. The proposed technique is proved by a series of experiments on various workload scenarios deployed in two distinct cloud providers. The final results produce that the proposed algorithm finds out the optimum combination of cloud resources that provides a balanced trade-off between deployment cost and QoS performance in relatively low runtime.

\section{TABLE I COMPARISON TABLE FOR DIFFERENT SCHEDULING TECHNIQUE}

\begin{tabular}{|c|c|c|c|c|}
\hline S.NO & Scheduling Techniques & Problem Statement & Result & Environment \\
\hline 1 & $\begin{array}{l}\text { Cloud-based workflow } \\
\text { Scheduling (CWSA) } \\
\text { Algorithm[1] }\end{array}$ & $\begin{array}{l}\text { Resource } \\
\text { Management in the } \\
\text { context of multi- } \\
\text { tenant cloud } \\
\text { computing }\end{array}$ & $\begin{array}{l}\text { Minimize the cost } \\
\text { of execution of the } \\
\text { workflows }\end{array}$ & Cloud Environment \\
\hline 2 & $\begin{array}{lr}\text { Cost and } & \text { energy aware } \\
\text { scheduling } & \text { algorithm } \\
(\mathrm{CEAS})[2] & \end{array}$ & $\begin{array}{l}\text { To execute the } \\
\text { deadline- } \\
\text { constrained } \\
\text { scientific workflows }\end{array}$ & $\begin{array}{l}\text { Minimize } \\
\text { execution cost of } \\
\text { workflow }\end{array}$ & Cloud Environment \\
\hline
\end{tabular}


International Journal of Trend in Scientific Research and Development (IJTSRD) ISSN: 2456-6470

\begin{tabular}{|l|l|l|l|l|}
\hline 3 & $\begin{array}{l}\text { Profit maximization } \\
\text { algorithm [4] }\end{array}$ & $\begin{array}{l}\text { Dynamically } \\
\text { scheduling task }\end{array}$ & $\begin{array}{l}\text { Increase profit and } \\
\text { throughput }\end{array}$ & Cloud data center \\
\hline $\mathbf{4}$ & $\begin{array}{l}\text { Improved ant colony } \\
\text { algorithm[3] }\end{array}$ & $\begin{array}{l}\text { Task-scheduling } \\
\text { problems }\end{array}$ & $\begin{array}{l}\text { To optimize } \\
\text { resource } \\
\text { Utilization }\end{array}$ & Cloud Environment \\
\hline $\mathbf{5}$ & $\begin{array}{l}\text { Multi-objective optimization } \\
\text { algorithm[6] }\end{array}$ & $\begin{array}{l}\text { Selection of optimal } \\
\text { cloud resource } \\
\text { To minimize cost } \\
\text { and maximize QoS }\end{array}$ & Cloud Environment \\
\hline $\mathbf{6}$ & $\begin{array}{l}\text { VM Profit Planning } \\
\text { algorithm[5] }\end{array}$ & $\begin{array}{l}\text { Virtual machine } \\
\text { scheduling }\end{array}$ & $\begin{array}{l}\text { To improve } \\
\text { resource utilization }\end{array}$ & Cloud data center \\
\hline
\end{tabular}

TABLE II COMPARISON ON SCHEDULING ALGORITHMS AND PERFORMANCE METRICS

\begin{tabular}{|c|c|c|c|c|c|c|}
\hline \multirow[t]{2}{*}{ Scheduling Techniques } & \multicolumn{6}{|c|}{ Performance Metrics } \\
\hline & 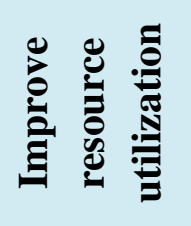 & 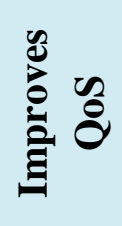 & 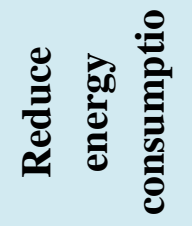 & 苋 & 苞 & 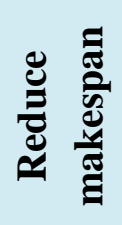 \\
\hline $\begin{array}{l}\text { Cloud-based workflow } \\
\text { Scheduling (CWSA) } \\
\text { Algorithm }\end{array}$ & - & - & - & $\checkmark$ & - & $\sqrt{ }$ \\
\hline $\begin{array}{l}\text { Cost and energy aware scheduling } \\
\text { algorithm (CEAS) }\end{array}$ & - & - & $\checkmark$ & $\checkmark$ & - & - \\
\hline Profit maximization algorithm & $\checkmark$ & - & - & - & - & - \\
\hline $\begin{array}{l}\text { Multi-objective } \\
\text { algorithm }\end{array}$ & - & $\checkmark$ & - & - & $\checkmark$ & - \\
\hline VM Profit Planning algorithm & $\checkmark$ & - & $\checkmark$ & - & - & - \\
\hline Improved ant colony algorithm & $\checkmark$ & - & - & - & $\checkmark$ & - \\
\hline
\end{tabular}

\section{CONCLUSION}

As the cloud computing technology is growing day by day many new challenges are rising. Task scheduling is one among them. In this paper an analysis of various existing scheduling techniques inthe areas like resource utilization, execution time and makspan, etc. ina cloud environment is done. The study also motivates for number of parameters which can be included in future. 


\section{REFERENCE}

1) Bhaskar Prasad Rimal, and MartinMaier, "Workflow Scheduling in Multi-Tenant Cloud Computing Environments" in IEEE Transactions on Parallel and Distributed Systems, Vol. 28, no. 1, pp. 290-304 Jan. 1, 2017.

2) Zhongjin Li, Jidong Ge, Haiyang $\mathrm{Hu}$, Wei Song, $\mathrm{Hao} \mathrm{Hu}$, and Bin Luo" Cost and Energy Aware Scheduling Algorithm for Scientific Workflow with Deadline Constraint in Clouds" in IEEE Transactions on Services Computing, Vol. 1, no. 99, PP.1-1, Sept. 25, 2015.

3) Lyon Zuo_y, Lei Shu_z, Shoubin Dong Y, Yuanfang Chen_, Li Yan_Guangdong, "A Multiobjective Hybrid Cloud Resource Scheduling Method Based on Deadline and Cost Constraints" in IEEE Access, Vol.1, no. 99, pp 1-1, Dec. 30, 2016.

4) Hat Yuan, Jing Bi, Wei Tan, and Bo $\mathrm{Hu}$ Li" Temporal Task Scheduling With Constrained Service Delay for Profit Maximization in Hybrid Clouds" IEEE Transactions on Automation Science and Engineering, vol. 14, no. 1, pp. 337 348, Jan. 2017.

5) Xibo Jin, Fa Zhang, Lin Wang, Songlin Hu, Biyu Zhou and Zhiyong Liu," Joint Optimization of Operational Cost and Performance Interference in Cloud Data Centers" in IEEE Transactions on Cloud Computing, Vol. 1, no. 99, pp. 1-1, June 25, 2015.
6) Seyedehmehrnaz Mireslami, Logan Rakai, Behrouz Homayoun Fa, and Mea Wang," Simultaneous Cost and QoS Optimization for Cloud Resource Allocation" in IEEE Transactions on Network and Service Management,vol. 14, no. 3, pp. 676 - 689,Sept. 2017

7) Barrie Sosinsky, "Defining Cloud Computing" in the Cloud Computing Bible, Indianapolis, Indiana: Wiley Publishing, Inc. 2011.

8) "Types of scheduling", https://www.go4expert.com/articles/types-ofscheduling-t22307/.

9) https://www.tutorialspoint.com/cloud_computing/ cloud_computing_overview.htm"cloud computing overview".

10) https://www.tutorialspoint.com/cloud_computing/ cloud_computing_infrastructure_as_a_service.htm 